\begin{tabular}{|c|l|}
\hline Title & Tailoring two-photon interference with phase dispersion \\
\hline Author(s) & Okamoto, Ryo; Takeuchi, Shigeki; Sasaki, Keiji \\
\hline Citation & $\begin{array}{l}\text { Physical Review. A, 74(1), 011801 } \\
\text { https://doi.org/_0.1103/PhysRevA.74.011801 }\end{array}$ \\
\hline Issue Date & 2006-07-10 \\
\hline Doc URL & http://hdl.handle.net/2115/33854 \\
\hline Rights & @2006 The A merican Physical Society \\
\hline Type & article \\
\hline File Information & PhysRevA_74_011801.pdf \\
\hline
\end{tabular}

Instructions for use 


\title{
Tailoring two-photon interference with phase dispersion
}

\author{
Ryo Okamoto, ${ }^{*}$ Shigeki Takeuchi, ${ }^{\dagger}$ and Keiji Sasaki ${ }^{\ddagger}$ \\ Research Institute for Electronic Science, Hokkaido University, Sapporo 060-0812, Japan
}

(Received 11 December 2005; published 10 July 2006)

\begin{abstract}
We present a complete analytical formalism of the effect of phase dispersion on the Hong-Ou-Mandel dip with cw pumping, including higher-order terms. We show that the Hong-Ou-Mandel dip is strongly modified by the frequency-dependent phase modulation and even becomes a bump when the phase dispersion is a special step function. We also show that the phase dispersion function can be fully reconstructed via Fourier transformation from the measurement result of the Hong-Ou-Mandel dip when the dispersion function is antisymmetric. An experimental demonstration of the proposed method using a bandpass filter as a test sample is also presented.
\end{abstract}

DOI: 10.1103/PhysRevA.74.011801

PACS number(s): 42.50.Dv, 03.67.-a, 42.50.Ct

The two-photon interference phenomenon with a beam splitter was first demonstrated by Hong, Ou, and Mandel (HOM) [1]. They used a pair of photons generated via spontaneous parametric downconversion (SPDC), in which a single photon of a pumping laser is converted into a pair of photons. These two photons were sent to a 50-50 beam splitter and were detected by single-photon counters after the beam splitter. They measured the number of coincidence events while scanning an optical delay added to the path of one of the photons, and found a sharp dip (a HOM dip), at which the delay was exactly zero. This two-photon interference phenomenon has become an important tool [2] in a variety of fields. In the field of quantum information, the phenomenon is used in Bell-state analyzers [3] for quantum teleportation [4] and in quantum gates $[5,6]$ and quantum filters [7] for linear optics quantum computation [8]. Recently, this phenomenon has come to be used in the field of optical tomography [9]. The effect of phase dispersion on two-photon interference, or the HOM dip, has also been attracting attention because the phase dispersion shows interesting characteristics of the phenomenon and of the downconverted photon pairs.

Franson [10] analyzed the effect of dispersion up to the second order and found that the width of the HOM dip and the degree of coincidence were unchanged, even when the dispersions experienced by each of the photons were similar. This phenomenon, called dispersion cancellation, was experimentally demonstrated by Steinberg et al. [11]. Later, Grice and Walmsley presented a detailed analysis of dispersion cancellation for photon pairs produced by ultrashort pulses [12]. They studied the effect of external dispersion up to the second order and found that the shape of the HOM dip is distorted and becomes asymmetric with a short (200 fs) pump pulse. Such an asymmetric HOM dip with femtosecond pulse laser pumping has been reported in the literature $[13,14]$. However, the effect of phase dispersion, including higher-order terms, on the shape of the HOM dip has not been analytically formulated [15] either for pulse laser pumping or for $\mathrm{cw}$ laser pumping.

\footnotetext{
*Electronic address: oka@es.hokudai.ac.jp

${ }^{\dagger}$ Electronic address: takeuchi@es.hokudai.ac.jp

ĐElectronic address: sasaki@es.hokudai.ac.jp
}

In this Rapid Communication, we present the complete analytical formalism of the effect of phase dispersion on the HOM dip with $\mathrm{cw}$ pumping, including higher-order terms. We show that the HOM dip is strongly modified by phase dispersion with higher-order terms and even becomes a "bump" when the phase dispersion is a special step function. Such analysis is not possible by previous methods. Under this condition, these two photons show a kind of antibunching, which is opposite to the phenomenon that "two photons always emerge together" [16] observed without phase dispersion. This type of quantum interference may be useful for quantum-information processing.

In addition, we found that the phase dispersion function can be fully reconstructed via Fourier transformation from the measurement result of the HOM dip when the dispersion function is antisymmetric. We also performed an experimental demonstration of this method using a bandpass filter as a test sample. We observed a strongly distorted HOM dip, even with cw pumping, when a test sample having higher-order phase dispersion was inserted in one of the photon paths. The reconstructed phase dispersion is compared to that calculated for an imaginary monolayer dielectric filter.

Suppose we have a sample material with a phase dispersion in the path of one of twin photons with a complex transmittance $\eta(\omega)=r(\omega) \exp [i \psi(\omega)]$, where $\omega, r(\omega)$, and $\psi(\omega)$ are the frequency, real transmittance, and phase shift, respectively, of the photons. Using the weight function $\phi\left(\omega_{1}, \omega_{0}-\omega_{1}\right)$ of the parametric fluorescence emitted at $\omega_{1}$ and $\omega_{0}-\omega_{1}$ with pump wavelength $\omega_{0}$, the wave function of the twin photons after passing through the material is

$$
|\Psi\rangle=\int_{0}^{\infty} \eta\left(\omega_{1}\right) \phi\left(\omega_{1}, \omega_{0}-\omega_{1}\right)\left|\omega_{1}\right\rangle\left|\omega_{0}-\omega_{1}\right\rangle d \omega_{1} .
$$

Note that in their pioneering paper Hong et al. assumed that $\phi\left(\omega_{1}, \omega_{0}-\omega_{1}\right)$ is real and that no phase dispersion existed in the path of the entangled photons [1], i.e., $\eta(\omega)=1$.

The joint probability of detection of the photons at both detectors at times $t$ and $t+\tau$ is given by

$$
\begin{aligned}
P_{12}(\tau, \delta \tau)= & K\left\{T^{2}|G(\tau)|^{2}+R^{2}|G(2 \delta \tau-\tau)|^{2}\right. \\
& \left.-R T\left[G^{*}(\tau) G(2 \delta \tau-\tau)+\text { c.c. }\right]\right\}
\end{aligned}
$$




$$
\begin{aligned}
G(\tau)= & \int_{-\infty}^{\infty} \eta\left(\omega_{0} / 2+\omega\right) \phi\left(\omega_{0} / 2+\omega, \omega_{0} / 2-\omega\right) \\
& \times \exp (-i \omega \tau) d \omega,
\end{aligned}
$$

where $K, \delta \tau, T$, and $R$ denote the constant characteristics of the detectors, the amount of optical delay inserted in one of the paths, and the transmittance and reflectance of the beam splitter, respectively. Here the integration region $\left(-\omega_{0} / 2\right.$ to $\infty$ ) of $\omega$ was replaced by $-\infty$ to $\infty$.

The coincidence count probability $N(\delta \tau)$ is obtained by integrating $P_{12}(\tau, \delta \tau)$ over the coincidence time window $T_{\mathrm{CC}}$ of a few nanoseconds, which is much longer than the coherent time of parametric fluorescence (typically a few picoseconds). In this case, the integration region of $\tau$ can be replaced by the region $-\infty$ to $\infty$. Thus, $N(\delta \tau)$ is given as follows:

$$
\begin{aligned}
N(\delta \tau)= & \int_{-\infty}^{\infty} K\left\{T^{2}|G(\tau)|^{2}+R^{2}|G(2 \delta \tau-\tau)|^{2}\right. \\
& \left.-R T\left[G^{*}(\tau) G(2 \delta \tau-\tau)+\text { c.c. }\right]\right\} d \tau .
\end{aligned}
$$

By substituting Eq. (3) in Eq. (4), we have

$$
N(\delta \tau)=K \int_{-\infty}^{\infty}|W(\tau, \delta \tau)|^{2} d \tau,
$$

where

$$
\begin{aligned}
W(\tau, \delta \tau)= & T \int_{-\infty}^{\infty} \eta\left(\omega_{0} / 2+\omega\right) \phi\left(\omega_{0} / 2+\omega, \omega_{0} / 2-\omega\right) \\
& \times \exp (-i \omega \tau) d \omega \\
& -R \int_{-\infty}^{\infty} \eta\left(\omega_{0} / 2+\omega\right) \phi\left(\omega_{0} / 2+\omega, \omega_{0} / 2-\omega\right) \\
& \times \exp [i \omega(\tau-2 \delta \tau)] d \omega .
\end{aligned}
$$

Using Parseval's theorem [17], Eq. (5) is transformed into the following equation:

$$
N(\delta \tau)=\frac{K}{2 \pi} \int_{-\infty}^{\infty}\left|\int_{-\infty}^{\infty} W(\tau, \delta \tau) \exp (-i \omega \tau) d \tau\right|^{2} d \omega .
$$

By substituting Eq. (6) in Eq. (7), we have

$$
\begin{aligned}
N(\delta \tau)= & 2 \pi K \int_{-\infty}^{\infty} \mid T \eta\left(\omega_{0} / 2-\omega\right) \phi\left(\omega_{0} / 2-\omega, \omega_{0} / 2+\omega\right) \\
& -R \eta\left(\omega_{0} / 2+\omega\right) \phi\left(\omega_{0} / 2+\omega, \omega_{0} / 2-\omega\right) \\
& \times\left.\exp (-i \omega 2 \delta \tau)\right|^{2} d \omega .
\end{aligned}
$$

Equation (8) indicates that the coincidence counting rate $N(\delta \tau)$ is symmetric when $\eta\left(\omega_{0} / 2+\omega\right)$ has only one- and two-order terms [18]. However, $N(\delta \tau)$ can be asymmetric when $\eta\left(\omega_{0} / 2+\omega\right)$ is not a real constant due to phase dispersion.

As an example of the case in which the effect of higherorder terms is significant, we calculated $N(\delta \tau)$ [Fig. 1(b)] for the phase dispersion $\psi(\omega)=\operatorname{Arg}[\eta(\omega)]$, which shows a sudden change in $\psi_{g}$ at the center wavelength $\omega_{0} / 2$ [Fig. 1(a)]. When $\psi_{g}=0$, i.e., phase dispersion does not exist, the graph
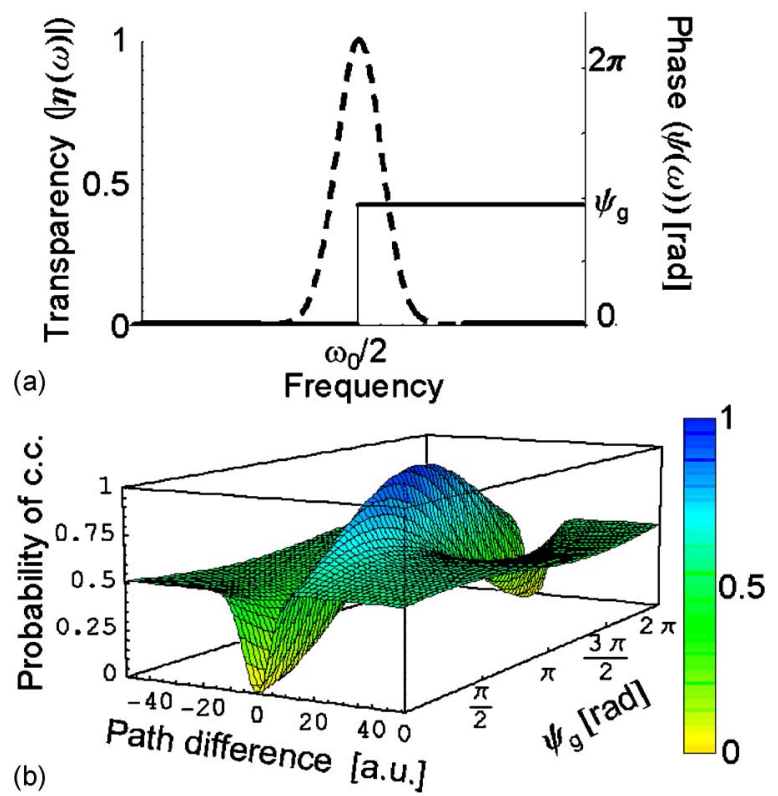

FIG. 1. (Color online) (a) Phase dispersion of the sample material inserted in the path of one of twin photons (solid line). The dotted line shows the absolute value of $\eta(\omega)$. (b) The calculated HOM dip for various phase gaps $\psi_{g}$ using the present theory. When $\psi_{g}=\pi$, the coincidence count probability shows a bump rather than the usual dip structure.

shows a typical HOM dip. However, as $\psi_{g}$ increases, the right side of the dip starts to rise and the shape of $N(\delta \tau)$ becomes asymmetric. When the phase gap $\psi_{g}=\pi$, the shape of $N(\delta \tau)$ becomes symmetric. However, the HOM dip is not a dip, but rather a bump [19]. In this condition, the coincidence probability becomes unity when the path length difference is zero. It means that these two photons are output from each output port one by one (antibunching), which is opposite to the phenomenon of two photons always emerging together [16] observed without phase dispersion. As $\psi_{g}$ increases further, the center part of the bump slides to the left and the shape gradually returns to the familiar HOM dip.

We have derived an exact formalism that shows how the HOM dip is distorted with arbitrary phase dispersion. Next, we show that the phase dispersion function can be estimated from the measured HOM dip.

By expanding and Fourier-transforming the right-hand side of Eq. (8), we have the following equation:

$$
\begin{aligned}
& \eta\left(\omega_{0} / 2-\omega\right) \eta^{*}\left(\omega_{0} / 2+\omega\right) \phi\left(\omega_{0} / 2-\omega, \omega_{0} / 2+\omega\right) \\
& \quad \times \phi^{*}\left(\omega_{0} / 2+\omega, \omega_{0} / 2-\omega\right) \\
& \quad=\frac{1}{2 R T} \int_{-\infty}^{\infty} \frac{N(\infty)-N(\delta \tau)}{2 \pi K} \exp (-i \omega 2 \delta \tau) d(2 \delta \tau),
\end{aligned}
$$

where

$$
\begin{aligned}
N(\infty) \equiv & 2 \pi K \int_{-\infty}^{\infty}\left\{\left|\operatorname{T\eta }\left(\omega_{0} / 2-\omega\right) \phi\left(\omega_{0} / 2-\omega, \omega_{0} / 2+\omega\right)\right|^{2}\right. \\
& \left.+\left|R \eta\left(\omega_{0} / 2+\omega\right) \phi\left(\omega_{0} / 2+\omega, \omega_{0} / 2-\omega\right)\right|^{2}\right\} d \omega
\end{aligned}
$$

corresponds to the coincidence count probability with sufficiently large $\delta \tau$. 


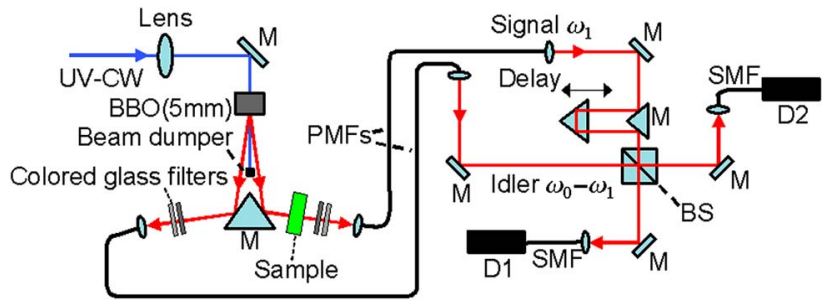

FIG. 2. (Color online) Schematic diagram of the experimental setup.

Suppose that we first perform a reference experiment without inserting the sample and obtain a coincidence rate (HOM dip) $N^{\prime}(\delta \tau)$. Using the measured $N^{\prime}(\delta \tau)$, we calculate $F^{\prime}(\omega)$, which is in the right-hand side of Eq. (9), from the measured coincidence rate $N^{\prime}(\delta \tau)$,

$$
F^{\prime}(\omega)=\frac{1}{2 R T} \int_{-\infty}^{\infty} \frac{N^{\prime}(\infty)-N^{\prime}(\delta \tau)}{2 \pi K} \exp (-i \omega 2 \delta \tau) d(2 \delta \tau)
$$

Next, we measure the coincidence rate $N(\delta \tau)$ with the sample inserted in the path of one of the twin photons and calculate $F(\omega)$ :

$$
F(\omega)=\frac{1}{2 R T} \int_{-\infty}^{\infty} \frac{N(\infty)-N(\delta \tau)}{2 \pi K} \exp (-i \omega 2 \delta \tau) d(2 \delta \tau) .
$$

Then, from Eqs. (9), (11), and (12) the complex transmittance of the sample can be estimated by the following equation:

$$
\eta\left(\omega_{0} / 2-\omega\right) \eta^{*}\left(\omega_{0} / 2+\omega\right)=\frac{F(\omega)}{F^{\prime}(\omega)} .
$$

The unknown phase dispersion $\psi(\omega)$ of the material can be found when we assume that $\psi(\omega)$ is antisymmetric with respect to $\omega_{0} / 2$ [20], as follows:

$$
\psi\left(\omega_{0} / 2-\omega\right)=\frac{1}{2} \operatorname{Arg}\left(\frac{F(\omega)}{F^{\prime}(\omega)}\right) .
$$

We performed a proof-of principle experiment using a narrow bandpass optical filter as a test sample. A schematic diagram of the experimental setup is shown in Fig. 2 [21]. A $\beta$-barium borate (BBO) crystal cut for the type-II twin-beam condition [22] was pumped by an argon ion laser at a wavelength of $351.1 \mathrm{~nm}$. The pump beam was focused in the BBO crystal using a convex lens to increase the photon flux [23]. The signal photon and idler photon were emitted at $702.2 \mathrm{~nm}$ and have horizontal and vertical polarization, respectively. As a test sample, a bandpass filter (center wavelength $702.2 \mathrm{~nm}$, full width at half maximum $0.3 \mathrm{~nm}$ ) was inserted before the fiber coupler. Each of the photons was then coupled to polarization-maintaining single-mode fibers (PMFs). Then, we twisted the PMF for the signal photon so that the polarization of the signal photon was changed to vertical. Thus, signal and idler photons with the same polarization (vertical polarization) were injected onto a 50-50

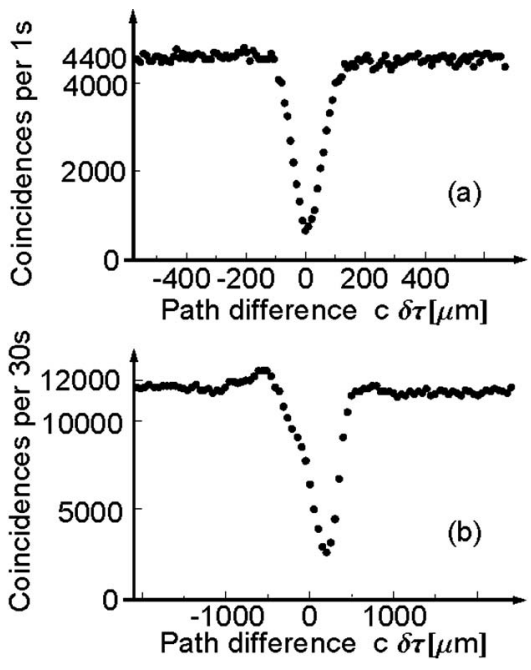

FIG. 3. Measured coincidence count rate without (a) and with (b) the sample. A narrow bandpass filter, the transmission spectrum of which is shown in the inset of Fig. 4, was used as a test sample.

beam splitter. The arrival timing of the two photons at the beam splitter was controlled using an optical delay. After the quantum interference that occurs at the beam splitter, the photons were reflected by mirrors [24], coupled into singlemode fibers, and counted by single-photon counters (SPCMAQ-FC, Perkin-Elmer).

The obtained HOM dip with and without the sample is shown in Figs. 3(b) and 3(a), respectively. The dip was distorted and became asymmetric when the narrow bandpass filter was inserted into one of the optical paths [Fig. 3(b)]. The coincidence count rate with sufficiently large $|\delta \tau|$ in Fig. 3(b) is smaller than that in Fig. 3(a) because some of the fluorescence was cut by the bandpass filter.

Next, we attempted to reconstruct the phase dispersion of the bandpass filter from the data shown in Figs. 3(a) and 3(b) [Eqs. (10)-(13)]. The reconstructed phase dispersion is shown as a dotted line in Fig. 4. The center frequency used for the reconstruction is $\omega_{0} / 2=2.68436 \times 10^{15}$. For comparison, a phase dispersion curve of an imaginary single-layer

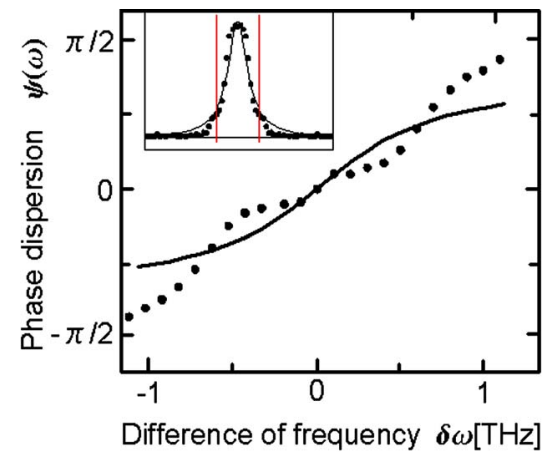

FIG. 4. (Color online) A reconstructed phase distribution (dotted line) of the sample using the data shown in Fig. 3. The solid line shows a calculated phase distribution for a monolayer filter, the transmission of which (inset, solid line) is similar to that of the actual sample (inset, dotted line). 
filter, the center wavelength and transmission spectrum (solid line in the inset of Fig. 4) of which are similar to those of the bandpass filter (dotted line in the inset of Fig. 4), is shown as the solid line in Fig. 4. Note that the measured transmission spectrum of the filter has a symmetric shape, suggesting that the phase dispersion curve satisfies the required assumptions for the estimation. The average inclination of the reconstructed phase dispersion is similar to that of the imaginary single-layer filter, but some inflection points that appear in the reconstructed data (dotted line) are not observed in the calculation for the imaginary filter (solid line). This may be because the actual filter has a complicated multilayer structure to suppress the leakage outside of the bandpass region, and so the reconstructed phase dispersion is different from the actual phase dispersion of the filter. This conjecture is supported by the fact that the HOM dip simulated using the calculated phase dispersion (solid line) was similar to the measured HOM dip [Fig. 3(b)] but did not show the bump in the left shoulder of the measured HOM dip.

We would like to thank Dr. J. O'Brien, Professor M. Kitano, and Professor N. Imoto for discussion. This study was supported in part by the Japan Science and Technology Corporation (JST), the Ministry of Internal Affairs and Communication (MIC), the Japan Science Promotion Society (JSPS), and the 21st Century COE Program.
[1] C. K. Hong, Z. Y. Ou, and L. Mandel, Phys. Rev. Lett. 59, 2044 (1987).

[2] For the test of this two-photon state, A. V. Burlakov, M. V. Chekhova, O. A. Karabutova, and S. P. Kulik, Phys. Rev. A 64, 041803(R) (2001).

[3] M. Michler, K. Mattle, H. Weinfurter, and A. Zeilinger, Phys. Rev. A 53, R1209 (1996).

[4] D. Bouwmeester, J.-W. Pan, K. Mattle, M. Eibl, H. Weinfurter, and A. Zeilinger, Nature (London) 390, 575 (1997).

[5] H. F. Hofmann and S. Takeuchi, Phys. Rev. A 66, 024308 (2002).

[6] T. C. Ralph, N. K. Langford, T. B. Bell, and A. G. White, Phys. Rev. A 65, 062324 (2002).

[7] H. F. Hofmann and S. Takeuchi, Phys. Rev. Lett. 88, 147901 (2002).

[8] E. Knill, R. Laflamme, and G. J. Milburn, Nature (London) 409, 46 (2001).

[9] M. B. Nasr, B. E. A. Saleh, A. V. Sergienko, and M. C. Teich, Opt. Express 12, 1353 (2004).

[10] J. D. Franson, Phys. Rev. A 45, 3126 (1992).

[11] A. M. Steinberg, P. G. Kwiat, and R. Y. Chiao, Phys. Rev. Lett. 68, 2421 (1992).

[12] W. P. Grice and I. A. Walmsley, Phys. Rev. A 56, 1627 (1997).

[13] M. Atatüre, A. V. Sergienko, B. M. Jost, B. E. A. Saleh, and M. C. Teich, Phys. Rev. Lett. 83, 1323 (1999).

[14] Y. H. Kim, V. Berardi, M. V. Chekhova, and Y. Shih, Phys. Rev. A 64, 011801(R) (2001).
[15] Some suggestions on the effects of higher-order terms of the phase dispersion are given in $[11,12]$.

[16] Z. Y. Ou, X. Y. Zou, L. J. Wang, and L. Mandel, Phys. Rev. A 42, 2957 (1990).

[17] A. Papoulis, The Fourier Integral and Its Applications (McGraw-Hill, New York, 1962).

[18] The first-order term simply shifts the entire dip, whereas the second-order terms are canceled and do not affect the shape of the dip (dispersion cancellation [10]).

[19] Note that the photons discussed here are unentangled in polarization and the origin of this bump is different from the ones with the photons entangled in polarization: P. G. Kwiat, A. M. Steinberg, and R. Y. Chiao, Phys. Rev. A 45, 7729 (1992); G. D. Giuseppe et al., ibid. 66, 013801 (2002); M. Atatüre et al., ibid. 66, 023822 (2002).

[20] This assumption is also applied in the estimation of phase dispersion using Kramers-Kronig relations.

[21] R. Okamoto, H. F. Hofmann, S. Takeuchi, and K. Sasaki, Phys. Rev. Lett. 95, 210506 (2005).

[22] S. Takeuchi, Opt. Lett. 26, 843 (2001).

[23] C. Kurtsiefer, M. Oberparleiter, and H. Weinfurter, Phys. Rev. A 64, 023802 (2001).

[24] In the actual experiment, we used polarizing beam splitters (PBSs) as mirrors for our convenience: since the input photons are vertically polarized and the polarization is not changed at the $50-50$ beam splitter, all of those photons were reflected by the PBS. 